\title{
The expression of RCAS1 and tumor infiltrating lymphocytes in patients with $\mathrm{T3}$ gastric carcinoma
}

\author{
Kenji Fukuda, Shunichi Tsujitani, Yoshiniko Maeta, Kenichi Yamaguchi, Masahide Ikeguchi, and \\ NobUaKi KaIBARA
}

First Department of Surgery, Faculty of Medicine, Tottori University, 36-1 Nishi-cho, Yonago 683-8504, Japan

\begin{abstract}
Background. This study aimed to assess the prognostic value of receptor binding cancer antigen expressed on SiSo cells (RCAS1) expression and host immune response in gastric carcinomas.

Methods. We investigated the relationship between RCAS1 expression, density of tumor-infiltrating lymphocytes (TIL), and clinicopathological findings in 129 patients with $\mathrm{T} 3$ gastric carcinoma who underwent curative surgery.

Results. RCAS1 immunoreactivity was detected in the membrane and cytoplasm of tumor cells. Positive immunoreactivity for RCAS1 was detected in 70 patients $(54.3 \%)$ and high expression levels of RCAS1 were found in 33 patients (25.6\%). The expression of RCAS1 significantly correlated with the histological type of carcinoma and lymph node metastasis. Apoptotic rates in TILs showed marginally higher significance in patients with high RCAS1 expression than in those with low expression. The 5-year survival rates were $77.9 \%$ in patients with low RCAS1 expression and $48.4 \%$ in those with high RCAS1 expression. Although there was no significant difference in survival between the patients with marked and slight infiltration of TILs, frequent apoptosis in TILs indicated significantly worse prognosis. Patients with low RCAS1 expression survived significantly longer than those with high RCAS1 expression, as did those patients with a high rate of apoptosis in TILs. Multivariate analysis revealed that RCAS1 expression, as well as tumor size and lymph node metastasis, was an independent prognostic factor.

Conclusions. The expression of RCAS1 was significantly correlated with poor prognosis in patients with $\mathrm{T3}$ gastric carcinoma. RCAS1 protein may play an important role in the evasion of tumor cells from immunological defense mechanisms in human gastric carcinoma.
\end{abstract}

Key words Gastric carcinoma-RCAS1·Tumor infiltrating lymphocyte $\cdot$ Immunohistochemistry $\cdot$ Prognostic factor

Offprint requests to: K. Fukuda

Received: March 13, 2002 / Accepted: August 26, 2002
Introduction

RCAS1 (receptor binding cancer antigen expressed on SiSo cells) was isolated as a cDNA encoding the antigen recognized by 22-1-1 antibody that was produced from mice immunized with the human uterine cervical adenocarcinoma cell line, SiSo [1,2]. The anti-RCAS1 monoclonal antibody strongly stained human uterine and ovarian carcinomas [3-5]. In nongynecological carcinomas, RCAS1 is also expressed in nonsmall-cell lung carcinomas, hepatocellular carcinomas, and squamous cell carcinomas of the skin [6-8]. In vitro studies of RCAS1 indicated that it might function as an apoptosisinducing factor, because binding between RCAS1 and its receptor induced apoptosis in receptor-expressing cells, including peripheral lymphocytes, such as T, B, and natural killer cells. Thus, RCAS1 was recognized to play a role in the immune evasion of tumor cells. Recent studies revealed that RCAS1 existed not only in neoplastic cells but also in cells in normal tissues, and that it controlled immune response [9-11].

Ropponen et al. [12] have reported that several human neoplasms contain many lymphocytes that infiltrate the center and periphery of the tumor, and they defined these lymphocytes as tumor-infiltrating lymphocytes (TILs). Moreover, the number of TILs was found to correlate with survival in colorectal and esophageal carcinoma patients $[12,13]$. Setälä et al. [14] investigated the prognostic value of various clinicopathological factors in 321 gastric carcinoma patients. In their study, the density of lymphocytes and plasma cells was not an independent prognostic predictor on multivariate analysis, despite its power on univariate analysis. On the other hand, gastric carcinoma with lymphoid stroma, infiltrated uniformly with an abundance of lymphocytes and plasma cells throughout the entire area of the tumor, is known to show an extremely high survival rate [15]. It has been reported that Fas and Fas ligand (FasL) induce apoptotic cell death of TILs in melanomas [16]. 
This apoptotic depletion of TILs by tumor cells has been suggested to cause tumor immune evasion [17-20]. However, data on the relationship between RCAS1 and the apoptosis of TILs in gastric carcinoma are scarce.

In the present study, the expression of RCAS1 and the numbers of TILs in advanced gastric carcinomas were examined. We attempted to elucidate the relationship between RCAS1, TILs, clinicopathological variables, and postoperative survival rates. Finally, the relationship between RCAS1 expression and the apoptosis of TILs was investigated to clarify the mechanisms of immune evasion in human gastric carcinoma.

\section{Patients and methods}

\section{Patients}

A total of 129 patients ( 75 men and 54 women; mean age $\pm \mathrm{SD}, 59 \pm 13$ years; range, 31-91 years) with primary gastric adenocarcinomas invading the serosa (T3 gastric carcinoma) underwent curative surgery between 1986 and 1992 at the First Department of Surgery, Tottori University Hospital. None of the patients had received preoperative adjuvant therapy. The clinicopathological findings were determined according to the Japanese classification of gastric carcinoma [21]. The criteria considered for curative resection were the complete removal of a primary gastric tumor, dissection of the regional lymph nodes, and no remaining macroscopic tumors. These patients had no metastases in the liver, or peritoneum, or in other distant organs at the time of surgery. No other previous or concomitant primary cancer was present. All had had either distal partial gastrectomy, proximal gastrectomy, or total gastrectomy with regional lymph node dissection to group 1 (D1), group 2 (D2), and group 3 (D3) excluding the paraaortic nodes, with curative intent. None of the resection margins were positive for tumors. Most patients received postoperative chemotherapy with the consecutive administration of uracil/tegafur, orally, at a dose of $300 \mathrm{mg}$ daily for 1 year after surgery. However, some patients refused or discontinued chemotherapy. The patients were periodically checked by diagnostic imagings (chest X-ray, double-contrast barium meal study, upper gastrointestinal fiberscopy, ultrasonography, and computed tomography) for early detection of recurrence. All of the patients were followed for at least 5 years.

\section{RCAS1}

For immunohistochemical analysis, streptavidin-biotin methodology (Histofine SAB-PO kit; Nichirei, Tokyo, Japan) was used for formalin-fixed and paraffin-embedded specimens. Sections $(4-\mu \mathrm{m})$ sections were cut from paraffin-embedded blocks. Tissue sections were deparaffinized and rehydrated in serial ethanol solutions. After repeated washing with phosphate buffered saline (PBS), the sections were treated with proteinase K (Dako, Glostrup, Denmark) for $3 \mathrm{~min}$. Endogenous peroxidase was blocked with $0.3 \%$ hydrogen peroxide in methanol for $10 \mathrm{~min}$. Tissue sections were then washed twice with PBS and preblocked for $10 \mathrm{~min}$ with $10 \%$ rabbit serum. Specimens were incubated overnight at $4{ }^{\circ} \mathrm{C}$ with anti-RCAS1 monoclonal antibody (dilution, 1:500; Medical and Biological Laboratories, Nagoya, Japan). Sections were washed three times in PBS and incubated with the secondary antimouse immunoglobulin $(\operatorname{IgG}+\operatorname{Ig} \mathrm{A}+\operatorname{IgM})$ conjugated with biotin (Nichirei) for $30 \mathrm{~min}$, followed by incubation with streptavidin-peroxidase complex (Nichirei) for $30 \mathrm{~min}$. After three additional washes in PBS, diaminobenzidine tetrahydrochloride working solution was applied. Finally, the slides were counterstained in hematoxylin. To examine the specificity of immunostaining, the primary antibody was replaced by normal mouse IgM. Tissue sections with more than 5\% reactive tumor cells were defined as positive. The staining pattern was classified as follows: negative, less than 5\% of reactive tumor cells; $1+$, weak (positive carcinoma cells were detected in $5 \%-25 \%$ ); $2+$, moderate (positive carcinoma cells were detected in $25 \%-50 \%$ ); and $3+$, strong (positive carcinoma cells were detected in $>50 \%$ ). All of the histological slides were examined by two observers (S.T. and Y.M.), who were unaware of the clinical data or the disease outcome. When the interpretation differed between the two observers, reevaluation was done, for a final decision on a conference microscope.

\section{TILs}

In addition to the examination of hematoxylin-and eosin-stained sections, lymphocyte infiltration into tumors was examined immunohistochemically using the anti-human T lymphocyte antibody (UCHL-1; Nichirei) in serial sections. UCHL-1 labeled resting T cells within both the CD4 and CD8 subsets and mature activated T cells. The immunostaining was performed as described above, except for the use of proteinase K. As a negative control, normal mouse IgG was used in place of UCHL1 at the same concentration. To identify nuclei with DNA strand break at a cellular level, anti-single-strand DNA (ssDNA) polyclonal antibody (dilution, 1:100; Dako Japan, Kyoto, Japan) was used. Immunostaining was done using the Envision+ kit (Dako, Carpinteria, CA, USA) by the dextran polymer method, according to the manufacturer's instructions. Border fields between gastric carcinoma and normal gland, which were observed under a microscope (magnification, $\times 200$ ), 
were randomly selected in each section. The degree of TIL infiltration was divided into the following two groups: marked infiltration, TILs infiltrated diffusely in the stroma of the tumor or around the tumor; and slight infiltration, TILs scattered in the stroma of the tumor or around the tumor. After detection of UCHL-1-positive TILs, at least five areas per slide were examined to count 1000 TILs and the number of ssDNA-positive apoptotic cells in serial sections. The results were defined as the apoptosis index (AI; the number of ssDNA-positive cells among 1000 TILs). The evaluation was done by two observers, as described above.

\section{Statistical analysis}

Statistical analysis included the standard $\chi^{2}$ test, MannWhitney $U$-test, and Kruskal-Wallis test. The survival rates were estimated by the Kaplan-Meier method, and statistically significant differences between survival curves were examined by the log-rank test. On multivariate analysis, independent prognostic factors were determined by the Cox proportional hazards model (StatView 5.0; Abacus Concepts, Berkeley, CA, USA). $P<0.05$ was regarded as statistically significant.

\section{Results}

\section{Immunohistochemistry}

RCAS1 protein existed both in the membrane and cytoplasm of tumor cells (Fig. 1a). Moreover, RCAS1 was very weakly but diffusely detected in the normal mu- cosal cells. RCAS1 staining in normal tissues was found more predominantly in the superficial layer than in the basal layer. The expression of RCAS1 in tumor cells was demonstrated in 70 patients $(54.3 \%)$. Following the previous report, we defined $25 \%$ of RCAS1-positivestaining cells in tumor cells as the cutoff value for low (negative $/ 1+$ ) and high $(2+/ 3+)$ expression. Of the 129 patients, 33 (25.6\%) showed high expression of RCAS1 protein $(2+$ in 18 and $3+$ in 15$)$, whereas $96(74.4 \%)$ demonstrated low expression (negative in 59 and $1+$ in 37).

\section{Clinicopathological analysis}

The relationship between RCAS1 expression and clinicopathological variables in T3 gastric carcinomas is shown in Table 1. There were significant correlations between RCAS1 expression and histological type of carcinoma or lymph node metastasis. High RCAS1 expression was more frequently observed in the undifferentiated type and in patients with $\mathrm{N} 2$ lymph node metastasis. In contrast, no significant correlation was detected between RCAS1 expression and age, sex, tumor size, or lymphatic-vascular space invasion.

\section{TILs}

Immunohistochemistry of TILs revealed that 74 patients had marked infiltration and 55 had slight infiltration. We investigated the relationship between infiltration and apoptosis in TILs (Fig. 1b). Tumors with slight TIL infiltration demonstrated significantly more
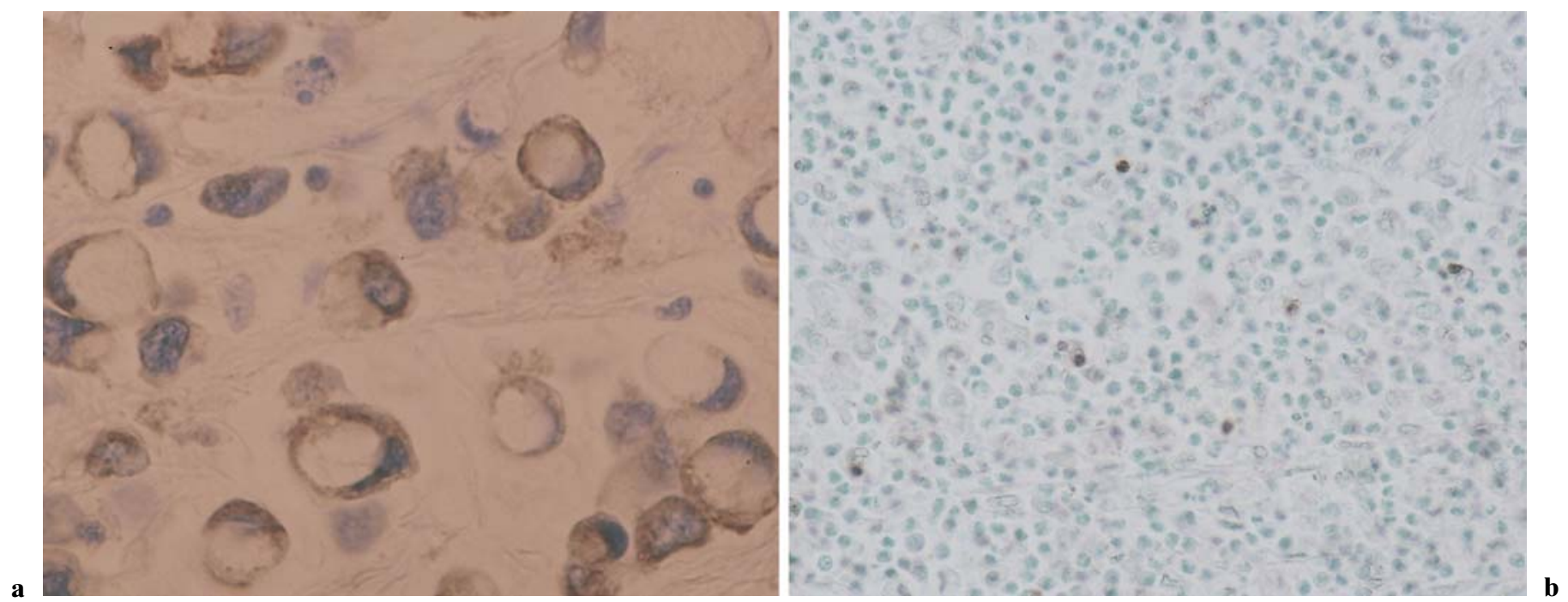

Fig. 1. a Receptor binding cancer antigen expressed on SiSo cells (RCAS1) immunostaining in gastric adenocarcinoma. RCAS1 was detected in the membrane and cytoplasm of cancer cells. b Apoptosis in tumor-infiltrating lymphocytes (TILs). a $\times 400$, b $\times 200$ 
Table 1. Association between RCAS1 expression and clincopathological variables

\begin{tabular}{lccc}
\hline & \multicolumn{2}{c}{ RCAS1 Expression } & \\
\cline { 2 - 3 } Variables & Low $(n=96)$ & High $(n=33)$ & $P$ value \\
\hline Age (years; mean \pm SD) & $59 \pm 13$ & $59 \pm 12$ & NS \\
Sex & 58 & 17 & NS \\
$\quad$ Male & 38 & 16 & NS \\
Female & $7.6 \pm 4.2$ & $7.5 \pm 3.0$ & $P<0.05$ \\
Size (cm; mean \pm SD) & 29 & 3 & \\
Histological type of tumor & 67 & 30 & \\
$\quad$ Differentiated & 54 & 16 & \\
$\quad$ Undifferentiated & 33 & 7 & NS \\
Lymph node metastasis & 9 & 10 & \\
$\quad$ N0 & 60 & 22 & NS \\
$\quad$ N1 & 36 & 11 & \\
$\quad$ N2 & & & \\
Lymphatic invasion & 62 & 20 & \\
$\quad$ Present & 34 & 13 & \\
$\quad$ Absent & & & \\
Venous invasion & & & \\
$\quad$ Present & & & \\
$\quad$ Absent & & &
\end{tabular}

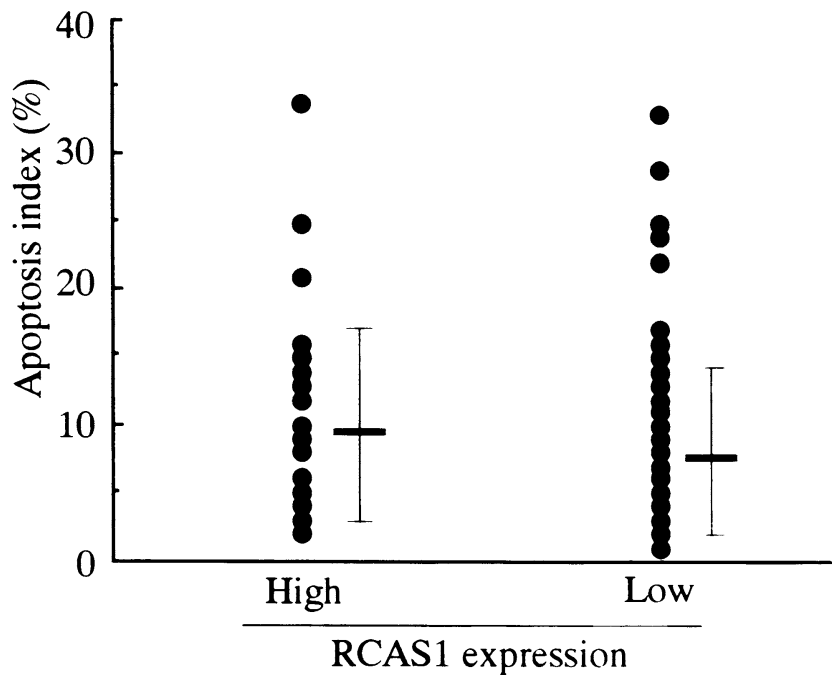

Fig. 2. The relationship of apoptosis in TILs and RCAS1 expression. Apoptosis of TILs was more frequent in tumors with high RCAS1 expression (apoptosis index; AI, $9.5 \pm 7.0$ ) than in those with low RCAS1 expression (AI, 7.5 \pm 6.2 ), with a marginally significant difference $(P=0.057)$

frequent apoptosis in TILs (AI, 9.2 \pm 7.2$)$ than those with marked TIL infiltration (AI, $7.1 \pm 5.6 ; P<0.05$, Student's $t$-test).

Furthermore, we analyzed the rate of apoptosis in TILs based on RCAS1 expression. Apoptosis of TILs was more frequent in tumors with high RCAS1 expression (AI, $9.5 \pm 7.0)$ than in those with low RCAS1 expression (AI, $7.5 \pm 6.2$ ), with a marginally significant difference ( $P=0.057$; Fig. 2$)$. Table 2 shows a detailed analysis of apoptosis in TILs. In patients with slight TIL infiltration, significantly higher rates of apoptosis were observed in RCAS1-positive tumors than in RCAS1negative tumors. However, the expression of RCAS1 did not affect the apoptosis in tumors with marked TIL infiltration. When tumors were $8 \mathrm{~cm}$ or more in diameter, there was no difference in the rate of apoptosis between tumors with high and low RCAS1 expression. In patients with tumors less than $8 \mathrm{~cm}$ in diameter, however, apoptosis was significantly more frequent in tumors with high RCAS1 expression than in those with low RCAS1 expression. Moreover, there was a marginally significant difference in the rate of apoptosis between tumors with high and low RCAS1 expression in patients with lymph node metastasis. In patients with no lymph node metastasis, however, there was no relationship between RCAS1 expression and the rate of apoptosis in TILs.

\section{Survival of patients}

The estimated 5-year overall survival rates of patients with low and high RCAS1 expression were $77.9 \%$ and $48.4 \%$, respectively (Fig. 3). Patients with high RCAS1 expression had significantly worse overall survival than those with low RCAS1 expression $(P<0.01)$. Table 3 shows the results of multivariate analysis for the 129 patients. The covariates included in the model were age and sex of patients, tumor size, histologic classification, lymph node metastasis, lymphatic invasion, venous in- 
Table 2. Infiltration and apoptosis in TILs

\begin{tabular}{|c|c|c|c|c|}
\hline & \multicolumn{2}{|c|}{ TIL infiltration } & \multirow[b]{2}{*}{$\begin{array}{c}\mathrm{AI} \\
(\text { mean } \pm \mathrm{SD})\end{array}$} & \multirow[b]{2}{*}{$P$ value } \\
\hline & $\begin{array}{l}\text { Marked } \\
\quad(n)\end{array}$ & $\begin{array}{l}\text { Slight } \\
(n)\end{array}$ & & \\
\hline \multicolumn{5}{|l|}{ RCAS1 } \\
\hline High & 17 & & $6.8 \pm 3.8$ & $P=0.68$ \\
\hline Low & 57 & & $7.1 \pm 6.1$ & \\
\hline High & & 16 & $12.4 \pm 8.5$ & $P<0.05$ \\
\hline Low & & 39 & $7.9 \pm 6.3$ & \\
\hline \multicolumn{5}{|c|}{ Large size tumor $(\geq 8 \mathrm{~cm})$} \\
\hline High RCAS1 & 8 & 7 & $7.9 \pm 5.3$ & $P=0.39$ \\
\hline Low RCAS1 & 19 & 23 & $7.5 \pm 4.6$ & \\
\hline \multicolumn{5}{|c|}{ Small size tumor $(<8 \mathrm{~cm})$} \\
\hline High RCAS1 & 9 & 9 & $10.9 \pm 8.1$ & $P<0.05$ \\
\hline Low RCAS1 & 38 & 16 & $7.4 \pm 7.2$ & \\
\hline \multicolumn{5}{|c|}{ Positive nodal involvement } \\
\hline High RCAS1 & 8 & 9 & $12.2 \pm 8.6$ & $P=0.058$ \\
\hline Low RCAS1 & 22 & 20 & $8.7 \pm 7.4$ & \\
\hline \multicolumn{5}{|c|}{ Negative nodal involvement } \\
\hline High RCAS1 & 9 & 7 & $6.6 \pm 3.1$ & $P=0.47$ \\
\hline Low RCAS1 & 35 & 19 & $6.5 \pm 4.8$ & \\
\hline
\end{tabular}

TILs, Tumor-infiltrating lymphocytes; AI, apoptosis index; $n$, number of patients

Table 3. Prognostic factors for overall survival selected by Cox's proportional hazards model

\begin{tabular}{lrccr}
\hline Variables & Coeff. & Coeff./SE & Hazard ratio & $P$ value \\
\hline Size $(8 \mathrm{~cm}$ or more $)$ & 0.992 & 2.713 & 2.521 & 0.0067 \\
$\begin{array}{l}\text { Lymph node metastasis } \\
\quad(\text { absent or present) }\end{array}$ & -0.811 & -2.412 & 0.444 & 0.0159 \\
$\begin{array}{l}\text { RCAS1 expression } \\
\quad \text { (low or high) }\end{array}$ & 0.992 & 2.971 & 2.696 & 0.0067 \\
\hline
\end{tabular}

Coeff., Coefficient; SE, standard error

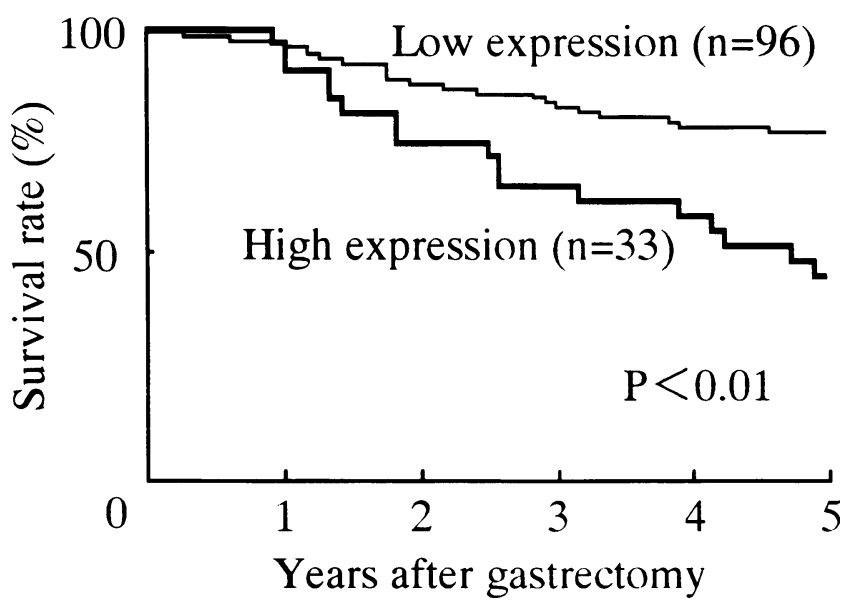

Fig. 3. Kaplan-Meier survival curves for patients with $\mathrm{T} 3$ gastric carcinoma who underwent curative gastrectomy. Prognosis of patients with high RCAS1 expression was significantly worse than that of those with low expression $(P<$ $0.01)$ vasion, and the RCAS1 expression. RCAS1 expression, as well as tumor size and lymph node metastasis, was recognized as an independent prognostic factor. Therefore, the clinical significance of RCAS1 expression was further analyzed based on the characteristics of tumors (Figs. 4, 5). The prognosis of patients with high RCAS1 expression was significantly poorer than that of those with low RCAS1 expression, when tumors were less than $8 \mathrm{~cm}$ in diameter or involved the regional lymph nodes. However, such a relationship was not observed in patients with tumor $8 \mathrm{~cm}$ or more in diameter or with no lymph node metastasis. There was a significant difference in survival between patients with high and low RCAS1 expression in patients with histologically undifferentiated type. The patients did not provide sufficient data for analysis, because of the small number of patients with high RCAS1 expression.

There was no significant difference in survival between patients with marked and slight infiltration of 

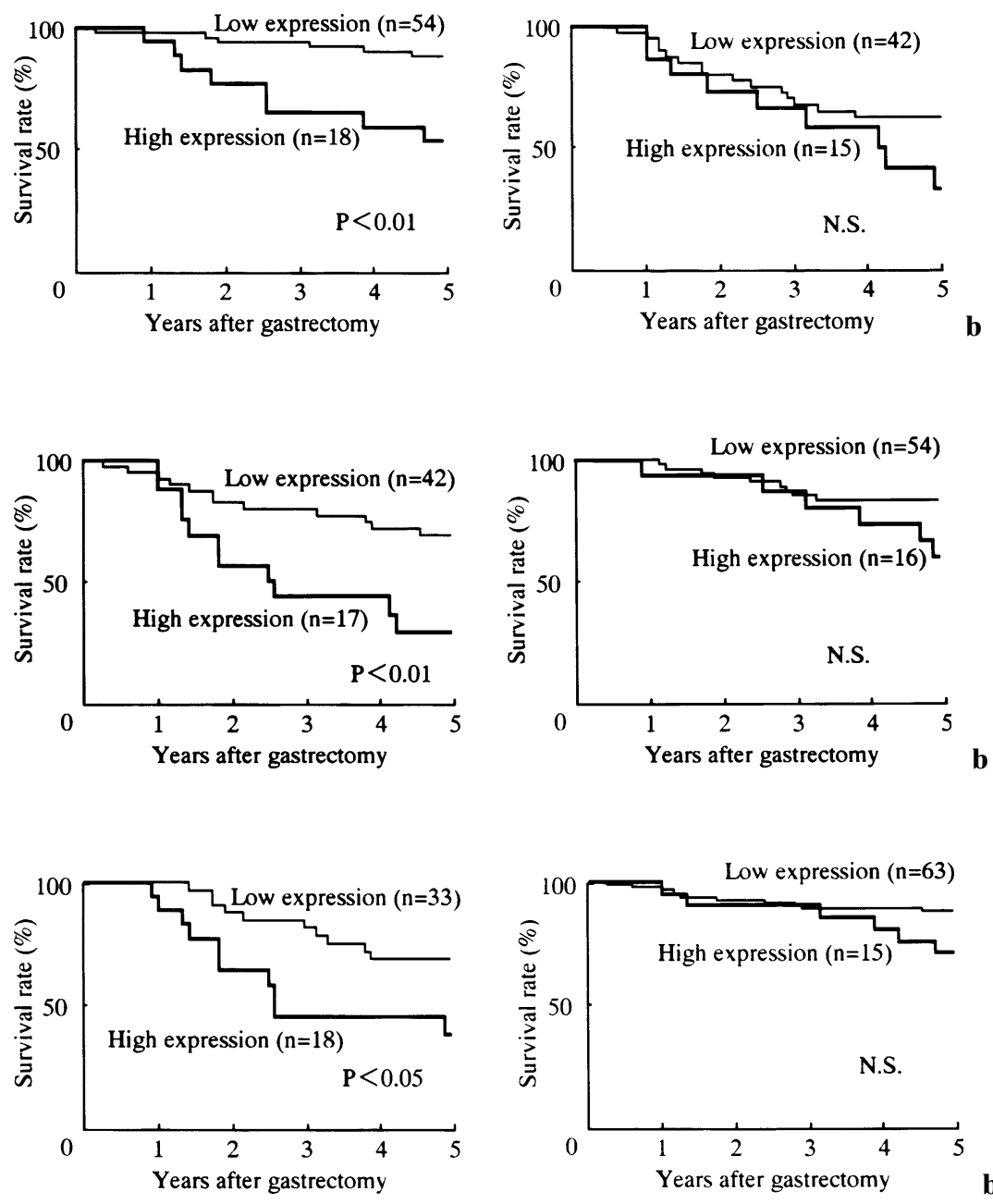

Fig. 4a,b. Kaplan-Meier survival curves for patients with T3 gastric carcinoma less than $8 \mathrm{~cm}$ in diameter (a), and $8 \mathrm{~cm}$ or more in diameter (b). In the patients with tumors less than $8 \mathrm{~cm}$ in diameter, the prognosis of patients with high RCAS1 expression was significantly poorer than that of those with low expression. N.S., Not significant

Fig. 5a,b. Kaplan-Meier survival curves for patients with T3 gastric carcinoma with lymph node metastasis (a), and no metastasis (b). In the patients with lymph node metastasis, the prognosis of patients with high RCAS1 expression was significantly poorer than that of those with low expression

Fig. 6a,b. Kaplan-Meier survival curves for patients in relation to RCAS1 expression and apoptosis of TILs. RCAS1 expression significantly correlated with poor prognosis in patients with TILs exhibiting frequent apoptosis (a), whereas there was no prognostic difference between RCAS1 expression levels in patients with a low rate of apoptosis in TILs (b)
TILs. However, the 5-year survival rates of patients with rare and frequent apoptosis in TILs were $76.5 \%$ and $55.7 \%$, respectively. Patients with rare apoptosis in TILs survived significantly longer than those with frequent apoptosis in TILs $(P<0.05)$. RCAS1 expression significantly correlated with poor prognosis in patients with TILs exhibiting frequent apoptosis $(P<0.05)$, whereas there was no prognostic difference between RCAS1 expression levels in patients with a low rate of apoptosis in TILs (Fig. 6).

\section{Discussion}

Nakashima et al. [2] found that RCAS1 inhibited the in vitro growth of its receptor-expressing cells and induced apoptotic cells. Moreover, when freshly isolated peripheral blood lymphocytes (PBLs) were co-cultured with RCAS1 protein, the growth of lymphocytes was suppressed. This inhibition of cell growth was more significant in CD3-positive cells than in normal PBLs. Given these facts, tumor cells were thought to attack the im- mune cells by expressing RCAS1. As for the function of RCAS1 to induce apoptosis in TILs in vivo, Iwasaki et al. [6] proved that the apoptosis rate of TILs in lung carcinoma patients with high RCAS1 expression was higher than that in those with low expression, by using the terminal deoxynucleotidyl transferase-mediated dUTP nick end labeling (TUNEL) method. Therefore, we investigated, in the present study, the relationship between RCAS1 expression and apoptosis in TILs in gastric carcinoma.

Immunohistochemical studies showed that various kinds of carcinoma expressed RCAS1 protein on the tumor cells at high rates; $87.5 \%$ in adenocarcinoma of the uterine cervix, $82.6 \%$ in invasive squamous cell carcinoma of the uterine cervix, $74.2 \%$ in lung carcinomas of various histologic types, and $73.8 \%$ in squamous cell carcinomas of the skin [3-8]. Recently, Kubokawa et al. [22] reported that RCAS1 was detected in $98.4 \%$ of gastric carcinomas. In the current series, however, RCAS1 was detected in $54.3 \%$ (70/129) of patients with gastric carcinoma invading the serosa. In addition, high expression was observed in $25.6 \%$ of patients. This 
difference may be due to differences in the methods of preservation of the specimen and antigen retrieval. Kubokawa et al. [22] used a universal immunoenzyme polymer (UIP) method without antigen retrieval, while a streptavidin-biotin (SAB) method with proteinase K antigen retrieval was used in the current study.

RCAS1 was also detected in normal mucosal cells of the stomach, especially in the superficial layer. This finding is consistent with the report by Kubokawa et al. [22]. It was reported that RCAS1 existed in normal uterine endometrial cells and in normal ciliated columnar epithelial cells of the lung at low levels $[3,6,11]$. Moreover, recent evidence shows that RCAS1, which is mainly produced by macrophages in hematopoietic tissue, may have a crucial role in regulating erythropoiesis by modulating apoptosis of erythroid progenitor cells [10]. It is necessary to investigate the biological functions of RCAS1 expression in nontumorous tissue.

TILs participate in tumor growth control not only through T-cell cytotoxity but also by producing soluble mediators [23]. The majority of TILs in human tumors consist of $T$ cells, suggesting an activation of the host defense mechanism [24]. Tumor-host interactions have been shown to play a significant role in predicting the disease outcome in several human tumors [24-26]. Furthermore, the human immune system can recognize many tumor-associated antigens, such as the MAGEfamily, p53, MUC-1, HER2/neu, and p21 ras [27-29]. Despite these antitumor mechanisms, the immune system often fails to inhibit tumor growth. In the current series, there was no significant difference in survival between patients with marked and slight infiltration of TILs. The reasons for this failure in tumor immunity might be insufficient expression of co-stimulatory or adhesion molecules by tumor cells, or defective processing and presentation of antigens on their cell surface [30-32]. Previous studies suggested that gastric carcinoma may require a greater number of TILs to overcome tumor growth than colorectal or esophageal carcinoma [12-15]. The current study was carried out to elucidate the multiple steps by which gastric carcinoma escapes from immunological defense mechanisms. Patients with frequent apoptosis in TILs had a significantly poorer prognosis than those with rare apoptosis in TILs.

Tumor cells may also escape from immune surveillance by expressing FasL or other molecules that induce apoptosis in activated T cells [15,33-35]. Similarly to the Fas-FasL system, RCAS1 inhibited the in vitro growth of lymphocytes expressing its receptor and induced apoptotic cell death, probably through the activation of interleukin (IL)-1 $\beta$ converting enzyme-like proteases [2]. In the present study, TILs in tumors with high RCAS1 expression demonstrated a higher AI than those with low expression, with a marginally significant difference. Apoptosis of TILs was more frequent in tumors with high RCAS1 expression than in those with low RCAS1 expression when tumors demonstrated slight TIL infiltration, smaller size, and lymph node metastases. These results suggest that RCAS1 expressed on tumor cells may induce apoptosis in lymphocytes positive for its receptor under certain conditions of tumor progression and thus contribute to tumor escape from the immune surveillance.

High RCAS1 expression was detected significantly more often in patients with $\mathrm{N} 2$ lymph node metastasis than in those with N0/N1 lymph node metastasis. This result was consistent with previous findings that RCAS1 expression was correlated with tumor progression [4,6$8,11]$. Multivariate analysis revealed that RCAS1, as well as tumor size and lymph node metastasis, was an independent prognostic factor. Moreover, patients with low RCAS1 expression survived significantly longer than those with high expression when apoptosis frequently occurred in TILs. Therefore, the clinical significance of RCAS1 expression may be related to tumor progression and TIL infiltration. The finding that RCAS1 expressed in tumor cells may not induce apoptosis in TILs in the situation of marked TIL infiltration suggests a way to overcome the immune escape by RCAS1.

In the current study, we investigated the expression of RCAS1 in gastric carcinomas with serosal invasion. RCAS1 expression correlated with lymph node metastasis and with the histologic type of tumor. Moreover, high RCAS1 expression induced marginally more frequent apoptosis of the TILs than low expression. Thus, it was suspected that tumor cells escaped from the immune system by expressing RCAS1, which induced apoptosis in the RCAS1-positive T cells. RCAS1 expression was an independent prognostic factor in the patients with advanced gastric carcinoma. In view of these findings, anti-RCAS1 antibody might be a useful tool for determining the prognosis or biological characteristics of advanced gastric carcinoma. Further investigation of the RCAS1 apoptotic TIL interaction might be valuable in developing novel immunotherapy for advanced gastric carcinomas.

\section{References}

1. Sonoda K, Nakashima M, Saito T, Amada S, Kamura T, Nakano $\mathrm{H}$, et al. Establishment of a new human uterine cervical adenocarcinoma cell line, SiSo, and its reactivity to anti-cancer reagents. Int J Oncol 1995;6:1099-104.

2. Nakashima M, Sonoda K, Watanabe T. Inhibition of cell growth and induction of apoptotic cell death by the human tumorassociated antigen RCAS1. Nature Med 1999;5:938-42.

3. Sonoda K, Nakashima M, Kaku T, Kamura T, Nakano H, Watanabe T. A novel tumor-associated antigen expressed in human uterine and ovarian carcinomas. Cancer 1996;77:1501-9.

4. Sonoda K, Kaku T, Kamura T, Nakashima M, Watanabe T, Nakano H. Tumor-associated antigen 22-1-1 expression in the 
uterine cervical squamous neoplasias. Clin Cancer Res 1998;4: 1517-20.

5. Kaku T, Sonoda K, Kamura T, Hirakawa T, Sakai K, Amada S, et al. The prognostic significance of tumor-associated antigen 221-1 expression in adenocarcinoma of the uterine cervix. Clin Cancer Res 1998;5:1449-53.

6. Iwasaki T, Nakashima M, Watanabe T, Yamamoto S, Inoue Y, Yamanaka $\mathrm{H}$, et al. Expression and prognostic significance in lung cancer of human tumor-associated antigen RCAS1. Int J Cancer 2000;89:488-93.

7. Noguchi K, Enjoji M, Nakamuta M, Nakashima M, Nishi H, Choi I, et al. Expression of tumor-associated antigen RCAS1 in hepatocellular carcinoma. Cancer Lett 2001;168:197-202.

8. Takahashi H, Iizuka H, Nakashima M, Wada T, Asako K, IshidaYamamoto A, et al. RCAS1 antigen highly expressed in extramammary Paget's disease and advanced stage squamous cell carcinoma of the skin. J Dermatol Sci 2001;26:140-4.

9. Ohshima K, Nakashima M, Sonoda K, Kikuchi M, Watanabe T. Expression of RCAS1 and FasL in human trophoblasts and uterine glands during pregnancy: the possible role in immune privilege. Clin Exp Immunol 2001;123:481-6.

10. Matsushima T, Nakashima M, Oshima K, Abe Y, Nishimura J, Nawata $\mathrm{H}$, et al. Receptor binding cancer antigen expressed on SiSo cells, a novel regulator of apoptosis of erythroid progenitor cells. Blood 2001;98:313-21.

11. Sonoda K, Kaku T, Hirakawa T, Kobayashi H, Amada S, Sasaki $\mathrm{K}$, et al. The clinical significance of tumor-associated antigen RCAS1 expression in the normal, hyperplastic, and malignant uterine endometrium. Gynecol Oncol 2000;79:424-9.

12. Ropponen KM, Eskelinen MJ, Lipponen PK, Alhava E, Kosma VM. Prognostic value of tumor-infiltrating lymphocytes (TILs) in colorectal cancer. J Pathol 1997;182:318-24.

13. Ikeguchi M, Saito H, Katano K, Tsujitani S, Maeta M, Kaibara N. Correlation between the lymphocytic infiltration of tumors and the proliferative activity of cancer cells from surgically treated esophageal carcinoma. Oncology 1997;54:311-7.

14. Setälä LP, Kosma V-M, Marin S, Lipponen PK, Eskelinen MJ, Syrjänen KJ. Prognostic factors in gastric cancer: the value of vascular invasion, mitotic rate and lymphoplasmacytic infiltration. Br J Cancer 1996;74:766-72.

15. Watanabe H, Enjoji M, Imai T. Gastric carcinoma with lymphoid stroma. Its morphologic characteristics and prognostic correlations. Cancer 1976;38:232-43.

16. Hahne M, Rimoldi D, Schroter M, Romero P, Schreier M, French LE, et al. Melanoma cell expression of Fas (Apo-1/CD95) ligand: implications for tumor immune escape. Science 1996;274:1363-6.

17. Nagata S. Fas ligand and immune evasion. Nature Med 1996;2: 1306-7.

18. Walker PR, Saas P, Dietrich PY. Role of Fas ligand (CD95L) in immune escape: the tumor cell strikes back. J Immunol 1997;158: 4521-4.

19. Strand S, Galle PR. Immune evasion by tumors: involvement of the CD95 (Apo-1/Fas) system and its clinical implications. Mol Med Today 1998;4:63-8.
20. Chappell DB, Restifo NP. T cell-tumor cell: a fatal interaction? Cancer Immunol Immunother 1998;47:65-71.

21. Japanese Gastric Cancer Association. Japanese classification of gastric carcinoma. 2nd English ed. Gastric Cancer 1998;1:1024.

22. Kubokawa M, Nakashima M, Yao T, Ito K, Harada N, Nawata $H$, et al. Aberrant intracellular localization of RCAS1 is associated with tumor progression of gastric cancer. Int J Oncol 2001;19:695700 .

23. Takagi S, Chen K, Schwarz R, Iwatsuki S, Herbermann RB, Whiteside TL. Functional and phenotypic analysis of tumorinfiltrating lymphocytes isolated from human primary and metastatic liver tumors and cultured in recombinant interleukin-2. Cancer 1989;63:102-11.

24. Underwood JCE. Lymphoreticular infiltration in human tumors: prognostic and biological implications. Br J Cancer 1974;30:53748.

25. Lipponen PK, Eskelinen MJ, Jahiainen K, Harju E, Terho R. Tumor infiltrating lymphocytes as an independent prognostic factor in transitional cell bladder cancer. Eur J Cancer 1992;29:6975.

26. Nakano T, Oka K, Takahashi T, Morita S, Arai T. Roles of Langerhans' cells and T-lymphocytes infiltrating cancer tissues in patients treated by radiation therapy for cervical cancer. Cancer 1992;70:2839-44.

27. Van den Eyndel BJ, Van der Bruggen P. T-cell defined tumor antigens. Curr Opin Immunol 1997;9:684-93.

28. Sahin U, Tureci O, Pfreundschuh M. Serological identification of human tumor antigens. Curr Opin Immunol 1997;9:70916.

29. Shichijo S, Nakao M, Imai Y, Takasu H, Kawamoto M, Niiya F, et al. A gene encoding antigenic peptides of human squamous cell carcinoma recognized by cytotoxic T lymphocytes. J Exp Med 1998;187:277-88.

30. Runger TM, Klein CE, Becker JC, Brocker, EB. The role of genetic instability, adhesion, cell mortality, and immune escape mechanism in melanoma progression. Curr Opin Immunol 1994; 6:188-96.

31. Edward M. Integrins and other adhesion molecules involved in melanocytic tumor progression. Curr Opin Immunol 1995;7:18591.

32. Ferrones S, Marincola FM. Loss of HLA class I antigens by melanoma cells: molecular mechanism, functional significance and clinical relevance. Immunol Today 1995;16:487-94.

33. Suda T, Nagata S. Purification and characterization of the Fasligand that induces apoptosis. J Exp Med 1994;179:873-9.

34. Strand S, Hofmann WJ, Hug H, Müller M, Otto G, Strand D, et al. Lymphocyte apoptosis induced by CD95 (APO-1/Fas) ligandexpressing tumor cells: a mechanism of immune evasion? Nature Med 1996;2:1361-6.

35. Gimmi CD, Morrison BW, Mainprice BA, Gribben JG, Boussiotis VA, Freeman GJ, et al. Breast cancer-associated antigen, DF3/MUC1, induces apoptosis of activated human T cells. Nature Med 1996;12:1367-70. 